\title{
NON-NEGATIVE SOLUTIONS FOR A CLASS OF RADIALLY SYMMETRIC NON-POSITONE PROBLEMS
}

\author{
ALFONSO CASTRO AND R. SHIVAJI \\ (Communicated by Walter D. Littman)
}

\begin{abstract}
We consider the existence of radially symmetric non-negative solutions for the boundary value problem

$$
\begin{gathered}
-\Delta u(x)=\lambda f(u(x)) \quad\|x\| \leq 1, x \in R^{N}(N \geq 2) \\
u(x)=0 \quad\|x\|=1
\end{gathered}
$$

where $\lambda>0, f(0)<0$ (non-positone), $f^{\prime} \geq 0$ and $f$ is superlinear. We establish existence of non-negative solutions for $\lambda$ small which extends some work of our previous paper on non-positone problems, where we considered the case $N=1$. Our work also proves a recent conjecture by Joel Smoller and Arthur Wasserman.
\end{abstract}

\section{INTRODUCTION}

Here we consider the existence of radially symmetric non-negative solutions for the boundary value problem

$$
\begin{gathered}
-\Delta u(x)=\lambda f(u(x)) \quad\|x\|<1, \quad x \in \mathbf{R}^{N}, \quad N \geq 2 \\
u(x)=0 \quad\|x\|=1
\end{gathered}
$$
where $\lambda>0$ and $f:[0, \infty) \rightarrow \mathbf{R}$ is such that $f^{\prime} \geq 0$ As is well documented, the study of $(1.1)-(1.2)$ is equivalent to the problem

$$
\begin{aligned}
-u^{\prime \prime}-(n / r) u^{\prime} & =\lambda f(u) ; \quad r \in(0,1) \\
u^{\prime}(0) & =0 \\
u(1) & =0
\end{aligned}
$$

where $n=N-1$. We will assume that

$$
\lim _{u \rightarrow+\infty}(f(u)) / u=+\infty \text {, i.e., } f \text { is superlinear, }
$$

Received by the editors October 26, 1987.

1980 Mathematics Subject Classification (1985 Revision). Primary 35J65, 34B15.

Key words and phrases. Non-negative solution, non-positone problem, singular o.d.e., Dirichlet problem.

Part of the first author's research was done while visiting Argonne National Laboratory.

The second author was supported by a Mississippi State University research initiation grant. 


$$
f(0)<0 \text { (non-positone) }
$$

and for some $k \in(0,1)$,

$$
\Lambda=\lim _{d \rightarrow+\infty}(d / f(d))^{N / 2}\{F(k d)-[(N-2) /(2 N)] d f(d)\}=+\infty
$$

where $F(x)=\int_{0}^{x} f(r) d r$.

If $f(0)>0$ (positone) and $\lambda>0$ small, it is known that (1.1)-(1.2) has two solutions: one near zero, the other bifurcating from infinity. However, the popular method of sub-super solutions used in positone problems seems rather difficult to apply when $f(0)<0$, since $v \equiv 0$ is no longer a sub-solution. In fact, it is a super solution. This is why we have been motivated to undertake this study. Our main result is given in Theorem 1.1.

Theorem 1.1. Under the above assumptions, there exists $\lambda_{0}>0$ such that if $0<\lambda<\lambda_{0}$, then (1.1)-(1.2) has a non-negative solution $u_{\lambda}$ such that $u_{\lambda}>0$ and decreasing on $[0,1]$ and $u_{\lambda}^{\prime}(1)<0$.

Castro and Shivaji [3] have made an extensive study of the one-dimensional problem $(N=1)$. Our proof of Theorem 1.1 is based on the shooting method. That is, to prove that (1.3)-(1.5) has a solution, we consider the problem (1.3)(1.4) subject to $u(0)=d$. By analyzing this problem depending on the parameter $d$, we show that for an adequate value of $d, u$ satisfies also (1.5). To prove Lemma 3.2 we use an identity of Pohozaev type (see $\$ 2$ ) used by Castro and Kurepa $[1,2]$ to study oscillatory solutions of other radially symmetric problems. For other applications and extensions of this type of identity, see Ni and Serrin [4].

Our work also proves a recent conjecture by Smoller and Wasserman [6]. In their paper they proved an existence result applicable to functions of the type $f(u)=u^{q}-\varepsilon$ where $\varepsilon>0,1<q<N /(N-2)$ and conjectured that an optimal result would be to extend it to $1<q<(N+2) /(N-2)$. In fact, our work includes this optimal result since if $f(u)=u^{q}-\varepsilon$ where $\varepsilon>0$, $1<q<(N+2) /(N-2)$ then (1.8) is satisfied with $k$ chosen larger than $[(q+1)(N-2) /(2 N)]^{(1 /(q+1))}$. Note here that if $q<(N+2) /(N-2)$ then $(q+1)(N-2) /(2 N)<\{(N+2) /(N-2)+1\}(N-2) /(2 N)=1$.

We will restrict our proofs in this paper to the case $N>2$. When $N=2$ the proof is easier along the same lines as in the case $N>2$.

\section{Preliminaries and Notations}

First of all we extend $f$ to $(-\infty, \infty)$ by defining $f(x)=f(0)$ for $x<0$. By (1.6) we see that $\lim _{d \rightarrow \infty} F(d)=\infty$. Hence (see (1.7)) there exist positive real numbers $\beta<\theta$ such that

$$
0=f(\beta)=F(\theta) .
$$

Since (see (1.8)) $\Lambda=\infty$, we see that there exists $\gamma>(\theta / k)$ such that

$$
2 N F(k d)-(N-2) d f(d) \geq 0 \quad \text { for } d \geq \gamma .
$$


Now for each real number $d$, the initial value (1.3), (1.4), $u(0)=d$ has a unique solution $u(t, d, \lambda)$. This solution depends continuously on $(d, \lambda)$ in the sense that if $\left\{\left(d_{n}, \lambda_{n}\right)\right\} \rightarrow(d, \lambda)$, then $\left\{u\left(, d_{n}, \lambda_{n}\right)\right\}$ converges uniformly to $u(, d, \lambda)$ on $[0,1]$. To see this, we observe that for each $(d, \lambda)$ the map

$$
u(s) \rightarrow d+\lambda \int_{0}^{s} t^{-n} \int_{0}^{t} r^{n}(-f(u(r))) d r
$$

defines a contraction on $C([0, \varepsilon], R)$ for $\varepsilon$ small enough.

Next given $d \in R, \lambda \in R$, we define

$$
\begin{gathered}
E(t, d, \lambda)=\frac{\left(u^{\prime}(t, d, \lambda)\right)^{2}}{2}+\lambda F(u(t, d, \lambda)), \\
H(t, d, \lambda)=t E(t, d, \lambda)+\frac{N-2}{2} u(t, d, \lambda) u^{\prime}(t, d, \lambda) .
\end{gathered}
$$

Multiplying (1.3) by $r^{N} u^{\prime}$ and integrating over $[\hat{t}, t]$, and then multiplying (1.3) by $r^{n} u$ and integrating over $[\hat{t}, t]$, we obtain

$$
\begin{aligned}
t^{N-1} H(t, d, \lambda)= & \hat{t}^{N-1} H(\hat{t}, d, \lambda)+\int_{\hat{t}}^{t} r^{n} \lambda[N F(u(r, d, \lambda) \\
& \left.-\frac{N-2}{2} f(u(r, d, \lambda)) u(r, d, \lambda)\right] d r .
\end{aligned}
$$

This identity is a form of "Pohozaev identity." For more details see Castro and Kurepa [1] and Pucci and Serrin [5].

Further, for $d \geq \gamma$ let $t_{0}:=t_{0}(d, \lambda)$ be such that $d \geq u\left(t_{0}, d, \lambda\right) \geq k d$ for all $t \in\left[0, t_{0}\right)$ and $u\left(t_{0}, d, \lambda\right)=k d$. Multiplying by $r^{n}(1.3)-(1.4)$ and $u(0)=d$ gives $u^{\prime}(t, d, \lambda)=-\lambda t^{-n} \int_{0}^{t} r^{n} f(u(r, d, \lambda)) d r$. Hence $-\lambda t f(k d) \geq$ $N u^{\prime}(t, d, \lambda) \geq-\lambda t f(d)$, and integrating on $\left[0, t_{0}\right]$ we have

$$
C_{1}\left\{d /(\lambda f(k d)\}^{1 / 2} \geq t_{0} \geq C_{1}\{d /(\lambda f(d))\}^{1 / 2}\right.
$$

where $C_{1}=\{(1-k) 2 N\}^{1 / 2}>0$. Also choosing $\hat{t}=0, t=t_{0},(2.6)$ gives

$$
\begin{aligned}
t_{0}^{n} H\left(t_{0}, d, \lambda\right) & =\lambda \int_{0}^{t_{0}} r^{n}\{N F(u(r, d, \lambda)) \\
& -[(N-2) / 2] f(u(r, d, \lambda)) u(r, d, \lambda)\} d r \\
& \geq \lambda \int_{0}^{t_{0}} r^{n}\{N F(k d)-[(N-2) / 2] f(d) d\} d r \\
& \geq \lambda\{N F(k d)-[(N-2) / 2] f(d) d\} t_{0}^{N} / N \\
& \geq \lambda\{N F(k d)-[(N-2) / 2] f(d) d\} \cdot\left\{C_{1}^{N} / N\right\} \cdot\{d /(\lambda f(d))\}^{N / 2} \\
& =C_{2} \lambda^{(1-N / 2)}\{F(k d)-[(N-2) /(2 N)] d f(d)\} \cdot\{d / f(d)\}^{N / 2}
\end{aligned}
$$

where $C_{2}=\left(C_{1}\right)^{N}>0$. 


\section{MAIN LEMMAS AND PROOF OF THEOREM 1.1}

Lemma 3.1. If $\lambda \in\left(0, \lambda_{1}:=N(\gamma-\beta) / f(\gamma)\right)$, then $u(t, \gamma, \lambda) \geq \beta$ for all $t \in$ $[0,1]$.

Proof. Let $t_{1}:=\sup \{t \leq 1 ; u(r, \gamma, \lambda) \geq \beta$, for all $r \in(0, t)\}$. Since $u^{\prime}(t, \gamma, \lambda)$ $=-\lambda t^{-n} \int_{0}^{t} s^{n} f(u(s, \gamma, \lambda)) d s, u$ is decreasing on $\left[0, t_{1}\right]$. Also if $\lambda \in\left(0, \lambda_{1}\right)$, $t \in\left[0, t_{1}\right]$, we have

$$
\left|u^{\prime}(t, \gamma, \lambda)\right| \leq \lambda t f(\gamma) / N<\gamma-\beta .
$$

Hence $u\left(t_{1}, \gamma, \lambda\right)>\gamma-(\gamma-\beta) t_{1}$. In particular, if $t_{1}<1$ this gives $u\left(t_{1}, \gamma, \lambda\right)>$ $\beta$ contradicting the definition of $t_{1}$. Thus $t_{1}=1$ and the lemma is proven.

Lemma 3.2. There exists $\lambda_{2}>0$ such that if $\lambda \in\left(0, \lambda_{2}\right)$, then $\{u(t, d, \lambda)\}^{2}+$ $\left\{u^{\prime}(t, d, \lambda)\right\}^{2}>0$ for $t \in[0,1], d \in[\gamma,+\infty)$.

Proof. Now for $t \geq t_{0},(2.6)$ and (2.8) gives

$$
\begin{aligned}
t^{n} H(t) \geq & \left.C_{2} \lambda^{(1-N / 2)}\{F k d)-[(N-2) /(2 N)] d f(d)\right\}\{d / f(d)\}^{n / 2} \\
& +\lambda \int_{t_{0}}^{t} r^{n}\{N F(u(r, d, \lambda))-[(N-2) / 2] f(u(r, d, \lambda)) u(r, d, \lambda)\} d r .
\end{aligned}
$$

Now by (1.8), our definition of $f(x)$ for $x<0$ and the fact that $f(0)<0$, there exists a constant $B<0$ such that $G(s)=N F(s)-[(N-2) / 2] f(s) s \geq B$ for all $s$. Further using (1.8), we may assume without loss of generality that $\gamma$ is large enough so that $\{F(k d)-[(N-2) /(2 N)] d f(d)\}\{d / f(d)\}^{N / 2} \geq 1$ for $d \geq \gamma$. Hence by (3.1) we have, for $t \in\left[t_{0}, 1\right]$,

$$
\begin{aligned}
t^{n} H(t) \geq & C_{2} \lambda^{(1-N / 2)}\{F(k d)-[(N-2) /(2 N)] d f(d)\} \cdot\{d / f(d)\}^{N / 2} \\
& +\lambda B\left\{t^{N}-t_{0}^{N}\right\} / N \\
\geq & C_{2} \lambda^{(1-N / 2)}+\lambda B / N \\
= & \lambda\left\{C_{2} \lambda^{-N / 2}+B / N\right\} .
\end{aligned}
$$

That is, there exists $\lambda_{2}$ such that for $\lambda \in\left(0, \lambda_{2}\right), H(t)$ (and hence $[u(t, \lambda, d)]^{2}$ $\left.+\left[u^{\prime}(t, \lambda, d)\right]^{2}\right)$ is positive for every $t \in[0,1]$ and every $d \in[\gamma,+\infty)$ and the lemma is proven.

Lemma 3.3. Given any $\lambda>0$, there exists $d>\gamma$ such that $u(t, d, \lambda)<0$ for some $t \in[0,1]$.

Proof. Let $\rho>0$ and $\omega$ be such that $\omega^{\prime \prime}+(n / t) \omega^{\prime}+\rho \omega=0, \omega(0)=1$, $\omega^{\prime}(0)=0$ and the first zero of $\omega$ is $\frac{1}{4}$. By (1.6), there exists $d_{0}(\lambda) \geq \theta / k$ such that if $x \geq d_{0}$ then

$$
(f(x) / x) \geq(\rho / \lambda) .
$$

Suppose now that for every $d>\gamma, u(t, d, \lambda) \geq 0$ for all $t \in[0,1]$. First we show that there exists $d_{1}(\lambda) \geq d_{0}(\lambda)$ such that for $d>d_{1}(\lambda)$ and $t_{1} \in(0,1]$

$$
\text { if } u^{\prime}\left(t_{1}, d, \lambda\right)=0 \text { then } u^{\prime \prime}\left(t_{1}, d, \lambda\right)<0 \text {. }
$$


In fact, let $d_{1}(\lambda)>d_{0}(\lambda)$ be such that if $d>d_{1}(\lambda)$ then $t^{n} H(t)>0$ for all $t \in\left[t_{0}, 1\right]$ (see (3.2)). Suppose there exists $t_{1} \in[0,1]$ with $u^{\prime}\left(t_{1}, d, \lambda\right)=0$, $u^{\prime \prime}\left(t_{1}, d, \lambda\right) \geq 0$. By $(1.3)$ we have $u\left(t_{1}, d, \lambda\right) \leq \beta$. Since $k d>\beta$ we have $t_{1}>t_{0}$. Thus $t_{1}^{n} H\left(t_{1}\right)=t_{1}^{N} \lambda F\left(u\left(t_{1}, d, \lambda\right)\right)<0$, which contradicts the definition of $d_{1}(\lambda)$. Thus (3.5) holds. By (3.5) we have that if $d>d_{1}(\lambda)$ and $t \in[0,1]$ then $u(t, d, \lambda) \cdot u^{\prime}(t, d, \lambda) \leq 0$. Hence, by (3.2) and the fact that $E(t, d, \lambda) \geq$ $\lambda F(k d)$ for $t \in\left[0, t_{0}\right]$, there exists $d_{2}(\lambda)>d_{1}(\lambda)$ such that for $d>d_{2}$

$$
E(t, d, \lambda) \geq \lambda F\left(d_{0}\right)+2 d_{0}^{2}
$$

for all $t \in[0,1]$. Now let $d>d_{2}$. Since $(d \omega)^{\prime \prime}+(n / r)(d \omega)^{\prime}+\rho(d w)=0$ and $u^{\prime \prime}+(n / r) u^{\prime}+\lambda(f(u) / u) u=0$, we get

$$
u(t)\left\{t^{n} v^{\prime}(t)\right\}-v(t)\left\{t^{n} u^{\prime}(t)\right\}=\int_{0}^{t} s^{n}\left\{\frac{\lambda f(u / s)}{u(s)}-\rho\right\} d s
$$

where $v=d \omega$. Hence if $u(t, d, \lambda) \geq d_{0}$ for all $t \in\left[0, \frac{1}{4}\right]$ then by (3.4) and facts that $v\left(\frac{1}{4}\right)=0, v^{\prime}\left(\frac{1}{4}\right)<0$ we obtain a contradiction to (3.6). Thus there exists $t^{*} \in\left(0, \frac{1}{4}\right)$ such that

$$
u\left(t^{*}, d, \lambda\right)=d_{0} .
$$

Also $u$ is decreasing on $\left(0, t^{*}\right)$ and (3.6) implies $u^{\prime}\left(t^{*}, d, \lambda\right) \leq-2 d_{0}$. Since we are assuming $u(t, d, \lambda) \geq 0$ for all $t \in\left(t^{*}, 1\right]$, we have $u^{\prime} \leq 0$ for $t \in\left[t^{*}, 1\right]$. Therefore $0 \leq u(t, d, \lambda) \leq d_{0}$ for $t \in\left(t^{*}, 1\right]$ and by (3.6) we have $u^{\prime}(t, d, \lambda) \leq$ $-2 d_{0}$ for all $t \in\left(t^{*}, 1\right]$. Hence integrating we have

$$
u\left(t^{*}+\frac{1}{2}, d, \lambda\right)-d_{0} \leq-2 d_{0} \cdot\left(\frac{1}{2}\right),
$$

that is, $u\left(t^{*}+\frac{1}{2}, d, \lambda\right) \leq 0$ where $t^{*}+\frac{1}{2}<1$, with $u^{\prime}\left(t^{*}+\frac{1}{2}, d, \lambda\right) \leq-2 d_{0}$. Thus there exists $T \in(0,1)$ such that $u(T, d, \lambda)<0$ which is a contradiction, hence the lemma is proven.

Proof of Theorem 1.1. Let $\lambda_{0}=\min \left\{\lambda_{1}, \lambda_{2}\right\}$ and $\lambda \in\left(0, \lambda_{0}\right)$. Let $\hat{d}(\lambda):=\hat{d}=$ $\sup \{d \in[\gamma,+\infty) ; u(t, d, \lambda) \geq 0$ for all $t \in[0,1]\}$. By Lemma 3.3 we have that $\hat{d}<+\infty$. Now we claim that:

(A) $u(1, \hat{d}, \lambda)=0$,

(B) $u(t, \hat{d}, \lambda)>0 \forall t \in[0,1)$,

(C) $u^{\prime}(1, \hat{d}, \lambda)<0$, and

(D) $u$ is decreasing on $[0,1]$.

Suppose there exists $T_{1}<1$ such that $u\left(T_{1}, \hat{d}, \lambda\right)=0$. Then Lemma 3.2 gives $u^{\prime}\left(T_{1} \hat{d}, \lambda\right) \neq 0$ and without loss of generality we can assume $u^{\prime}\left(T_{1}, \hat{d}, \lambda\right)<$ 0 . Thus there exists $T_{2} \in\left(T_{1}, 1\right)$ such that $u\left(T_{2}, \hat{d}, \lambda\right)<0$, a contradiction to the definition of $\hat{d}$. This proves (B). That is, $u(1, \hat{d}, \lambda) \geq 0$. Suppose $u(1, \hat{d}, \lambda)>0$. Then there exists $\eta>0$ such that $u(t, \hat{d}, \lambda) \geq \eta$ for all $t \in[0,1]$. Thus there exists $\delta>0$ such that $u(t, \hat{d}+\delta, \lambda) \geq \eta / 2$ for all $t \in[0,1]$, which contradicts the definition of $\hat{d}$. Hence $u(1, \hat{d}, \lambda)=0$ and 
(A) is proven. Finally (C) follows from Lemma 3.2 and (D) follows by Gidas, $\mathrm{Ni}$ and Nirenberg [7].

Remark. Unlike the case $N=1$ (see Castro and Shivaji [1987, Theorem 1.2]), for $N \geq 2$ the problem (1.1)-(1.2) does not have non-negative solutions with interior zeros. This follows because if there exists $t_{0} \in(0,1)$ for which $u^{\prime}\left(t_{0}\right)=$ $u\left(t_{0}\right)=0$ then $E\left(t_{0}\right)=0$. By (1.3) we obtain $d E / d t=-n\left(u^{\prime}\right)^{2} / t \leq 0$. But $E(1) \geq 0$. Thus $E=0$ for all $t \in\left[t_{0}, 1\right]$ which is possible only if $u^{\prime}=0$ and hence $u=0$ for all $t \in\left[t_{0}, 1\right]$. But from (1.3) we see that this is impossible with $f(0)<0$.

\section{REFERENCES}

1. A. Castro and A. Kurepa, Energy analysis of a nonlinear singular differential equation and applications, Rev. Colombiana Mat. (to appear).

2. A. Castro and A. Kurepa, Infinitely many solutions to a superlinear Dirichlet problem in a ball, Proc. Amer. Math. Soc. 101 (1) (1987), 57-64.

3. A. Castro and R. Shivaji, Non-negative solutions for a class of non-positone problems, Proc. Royal Soc. Endinburgh Sect. A 108, (1988) 291-302.

4. W. Mo Ni and J. Serrin, Non-existence theorems for quasi linear partial differential equations, Rend. Circ. Mat. Palermo (5) Suppl (1986).

5. P. Pucci and J. Serrin, A general variational identity, Indiana Univ. Math. J. 35 (3) (1986), 681-703.

6. J. Smoller and A. Wasserman, Existence of positive solutions for semilinear elliptic equations in general domains, Arch. Rational Mech. Anal. 98 (3) (1987), 229-249.

7. B. Gidas, W. M. Ni and L. Nirenberg, Symmetry and related properties via the maximum principle, Comm. Math. Phys. 68 (1979), 209-243.

Department of Mathematics, University of North Texas, Denton, Texas 76203-5116

Department of Mathematics, Mississippi State University, Mississippi State, Mississippi 39762 